\title{
Circle Sector Expansions for On-Line Exploration
}

\author{
Sven Rönnbäck Tomas Berglund \\ Division of EISLAB \\ Department of Computer Science \\ and Electrical Engineering \\ Luleå University of Technology \\ SE-97187 LULEÅ, SWEDEN \\ http://www.ltu.se/csee
}

Håkan Fredriksson Kalevi Hyyppä

\begin{abstract}
A novel and effective method denoted circle sector expansion $(C S E)$ is presented that can be used to generate reduced Voronoi diagrams. It is intuitive and can be used to efficiently compute possible paths for a vehicle. The idea is to model free space instead of the features in the environment. It is easy to implement and can be used while a vehicle moves and collects new data of its surrounding. The method is directly applicable and has properties for fast computations of safety margins while at the same time having low complexity.

We have successfully implemented the algorithm and its methods and performed real-life tests using an autonomous wheelchair equipped with a range scanning laser, a rate gyro, and wheel-encoders. Tests showed good results for supporting the use of CSE. The results are applicable for example to improve assistive technology for wheelchair users.
\end{abstract}

\section{INTRODUCTION}

${ }^{1}$ The problem of creating a Voronoi diagram from a set of $N$ points has been widely studied. In 1987 the Fortune sweepline Voronoi algorithm was published. It computes the Voronoi diagram in $O(N \log N)$ time [1]. The sweep-line algorithm creates a complete Voronoi diagram and the diagram needs to be reduced for efficient use in mobile navigation [2], [3], [4]. The generalized form of Voronoi diagram calculation requires that the environment is parameterized in form of polygon lines [5], [6]. The problem of identifying polygon lines from range data has lots of association problems. Voronoi skeletons are interesting for navigation and can be used for localization [7], [8], [9], [10], [11].

The mentioned methods either require as input some precalculated parametrization of the environment, grid based methods, or they compute a full Voronoi diagram. The latter is a non-compact representation not always suitable nor easily used for practical purposes.

We present a novel and effective on-line method for exploring an environment. It is denoted circle sector expansion (CSE) and can be used while a vehicle is moving and at the same time gathers new information from its surrounding. It is [12] a wavefront based method and CSE is an iterative process where consecutive expanded circle sectors are bounded by collected range data, Figure 1. The polygonal connected origins of the circle sectors build an approximate reduced Voronoi diagram.

\footnotetext{
${ }^{1}$ This work is supported by the EU program Interreg IIIA Nord
}

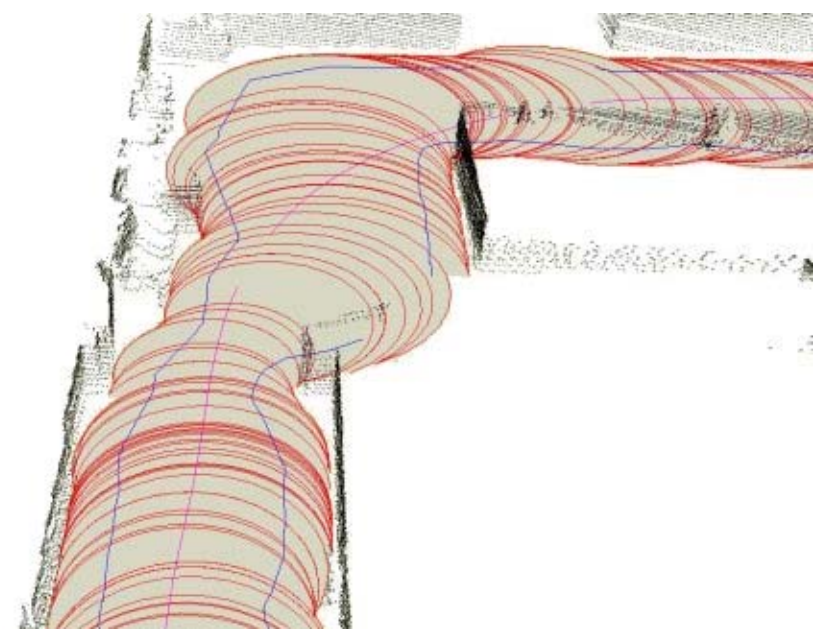

Fig. 1. The circle sector expansion method (CSE) is used to find available free-space for an autonomous wheelchair equipped with a ladar. The consecutive circle sectors spans up the available free-space. Five interesting things are found in the figure: 1) Safety boundaries created from the circle sectors are the polygons that follows the contours of the walls. 2) An estimated trajectory of the wheelchair is visible as the smooth line plotted on the floor. 3) A vertical pipe is mounted on the wall near the lower right corner. 4) The places where the contour polygons are discontinuous are gateways which are wide enough for the wheelchair to traverse. 5) Near the upper left corner a bench and some boxes are visible in the laser data.

Instead of focusing on the geometry and properties of obstacles, the CSE method puts the focus on free space and places and directions that the vehicle is able to traverse.

It can be used in an unknown environment and can handle difficult passages as well as discover possible paths from a certain location based on vehicle width. The length of a circle sector chord decides whether it is possible or not for the vehicle to reach a certain position in front of it.

The consecutive circle sectors are also used to rapidly calculate safety margins such that the vehicle does not touch obstacles.

\section{THE CIRCLE SECTOR EXPANSION METHOD}

It is a novel approach and can be used for realtime obstacle avoidance and to compute safety margins for a robot in a two dimensional workspace. 
The fundamental idea of the method is to find the bounding point for an expanding circle sector, visible in Figure 2.

A bounding point splits the current sector into two new circle sectors and the process is repeated.
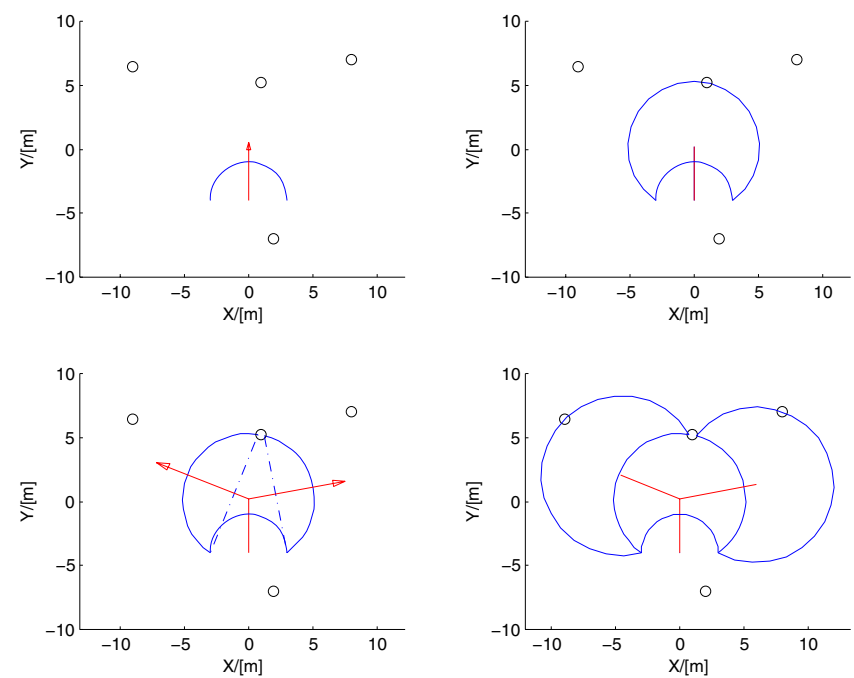

Fig. 2. Example of an expansion sequence. Four points are represented as the small circles. 1) The initial condition before expansion where the arrow indicates the direction of expansion. 2) The forward expansion is bounded by a point. 3) The expanded sector is divided into two new sectors. The new expanding directions are marked with arrows. 4) The result after three sector expansions.

If no bounding point is found the radius is undefined; in such a case the radius is set to a fixed value.

Each circle sector indicates a direction to free space, see Figure 1. If the length of a circle sector chord is larger than the width of the vehicle plus the safety margin, it can be part of a possible path for the vehicle. The output from the circle sector expansion is consecutive circle sectors.

\section{A. The CSE expansion process}

Figure 3 shows more in detail an example of an expansion sequence where three points, marked as 3,4 and 5 are present in front of the starting sector. The expansion process starts at the sector denoted $c 1$. The vehicle is represented as the thick circle. The thick arrows mark the expanding directions of the circle sectors. The dashed circle arcs are possible circle sectors candidates. Points are present inside the dashed circles and therefor they are disqualified as circle sectors for the expansion process. The largest expanded empty circle sector is always selected in each iteration. When the largest chord is selected we get the expansion sequence $c 1, c 2, c 3$ and $c 4$. The connected chords generates the Delaunay triangles.

\section{B. Circle sector parameters}

Circle sector $\sec (k)$ is defined by five parameters, $\sec (k)=$ $\left\{x_{c}(k), y_{c}(k), r_{c}(k), \theta_{c}(k), \beta_{c}(k)\right\}$, where $k$ is the iteration step. They are visualized in Figure 4 , where $\theta_{c}(k)$ is the heading of the circle sector, $\beta_{c}(k)$ is the sector angle, $r_{c}(k)$ is the radius, and $z_{c}(k)$ is the origin of the circle.

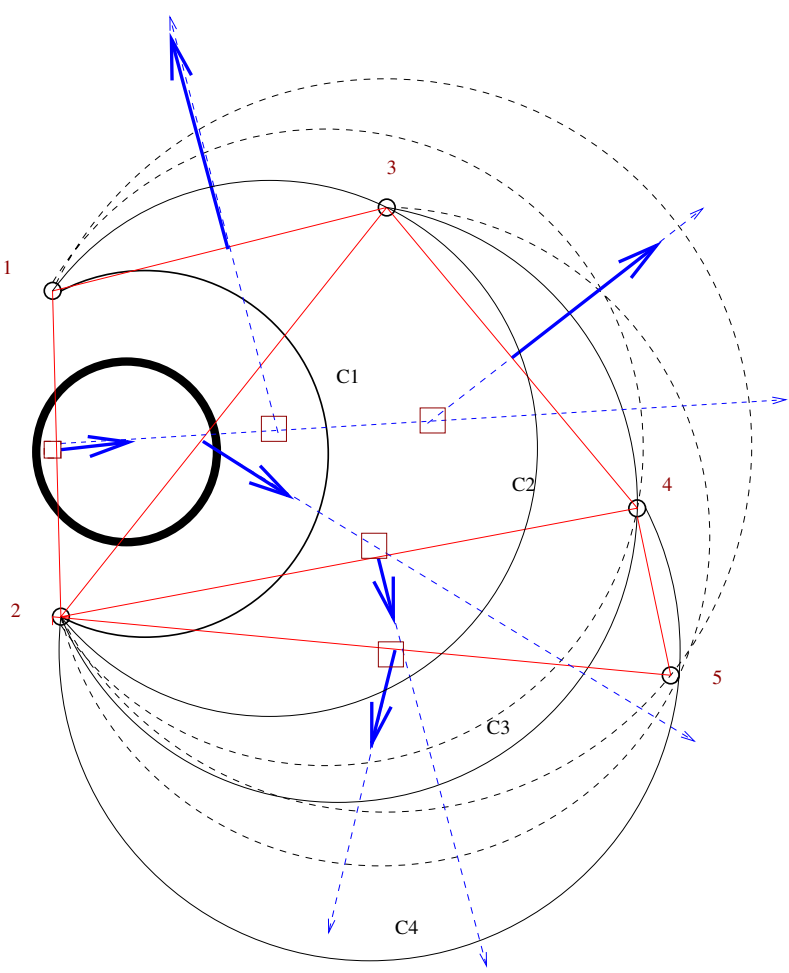

Fig. 3. An illustration of an expansion sequence in an environment with three points present to the right of the starting chord defined by the points 1 and 2 . The solid arrow marks the expanding directions of the circle sectors. Chord 4-5 is to short for the vehicle to traverse. The dashed straight lines build the Voronoi graph. The squares are origins for the expanding circle sectors.

The point that lies on the circle arc and bounds the circle sector is the complex number $z_{2}(k)$. To calculate the heading of the two new circle sectors we first perform a parametrization, called $\lambda_{c}(k)$, of point $z_{2}(k)$ along the sector arc,

$$
\lambda_{c}(k)=\frac{1}{2}+\frac{\angle\left[\left(z_{2}(k)-z_{c}(k)\right)\right]-\theta_{c}(k)}{\beta_{c}(k)} .
$$

The notation $\angle z$ returns the angular value of the complex number $z$.

Since point $z_{2}(k)$ splits the circle sector into two new sectors, $\lambda_{c}(k) \in(0,1)$. If $\lambda_{c}(k) \equiv 0.5$ the new sectors are equal in size. If $\lambda_{c}(k)<0.5$ the left sector is larger than the right one. The sector angles for the new circle sectors are

$$
\beta_{c R}(k)=\beta_{c}(k) \lambda_{c}(k),
$$

and

$$
\beta_{c L}(k)=\beta_{c}(k)\left(1-\lambda_{c}(k)\right) .
$$

The heading angles for the sectors expanding in the right and left direction are described by equation

$$
\theta_{c R}(k)=\angle\left[z_{2}(k)-z_{c}(k)\right]-0.5 \beta_{c R}(k)
$$

and

$$
\theta_{c L}(k)=\angle\left[z_{2}-z_{c}(k)\right]+0.5 \beta_{c L}(k),
$$

respectively. The heading angles are expressed in vector form as $e^{i \theta_{c}(k)}$. 


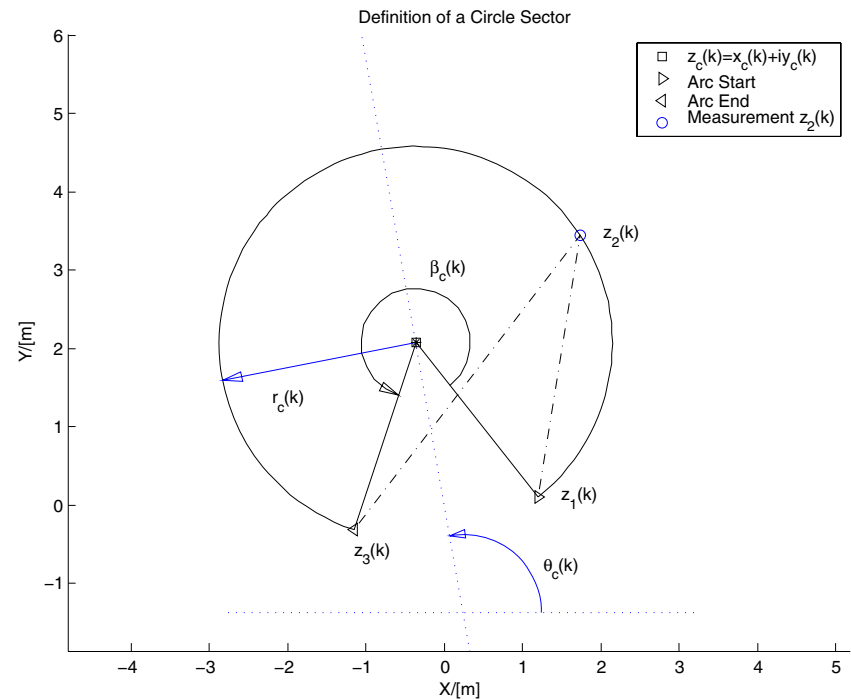

Fig. 4. A circle sector with sector angle $\beta_{c}(k)$, radius $r_{c}(k)$, heading angle $\theta_{c}(k)$ and circle origin $z_{c}(k)$. The laser measurement $z_{2}(k)$ divides the sector into two new sectors. The dashed lines between $z_{1}(k), z_{2}(k)$ and $z_{2}(k), z_{3}(k)$ are the chords for the new circle sectors.

1) Given a desired target position: Given a target position $z_{t}$ the expansion process can proceed to the left or the right relative to split point $z_{2}(k)$. A bearing angle is calculated relative to the vector that points towards the bounding measurement $z_{2}(k)$

$$
\gamma_{t}(k)=\angle\left[\left(z_{t}-z_{c}(k)\right)\left(\overline{z_{2}(k)-z_{c}(k)}\right)\right]
$$

If $\gamma_{t}(k)$ is positive the left sector is selected.

\section{Calculation of the circle sector parameters}

The output of the CSE method are consecutive circle sectors. Below follows a description on how to compute the parameters of a circle sector.

A circle sector with the parameters $z_{c}, r_{c}, \beta_{c}$ and $\theta_{c}$ is bounded and defined by the complex numbers $z_{1}, z_{2}$ and $z_{3}$ shown in Figure 5.

The origin, $z_{c}$, is calculated by translating the chord mid point, $\left(z_{1}+z_{3}\right) / 2$, along the vector that is orthogonal to the chord and points towards $z_{2}$.

The circle sector is undefined if $z_{1}, z_{2}$, and $z_{3}$ lie on a line. The inscribed angle $\gamma_{c}$ is found by taking the argument of the complex number $z_{v}$, i.e. $\gamma_{c}=\angle z_{v}$, where the complex number $z_{v}$ is calculated with

$$
z_{v}=\left(z_{1}-z_{2}\right) \overline{\left(z_{3}-z_{2}\right)},
$$

where $\overline{\left(z_{3}-z_{2}\right)}$ is the complex conjugate of $\left(z_{3}-z_{2}\right)$.

The expression for the circle sector origin $z_{c}$ is

$$
z_{c}=\frac{\left(z_{1}+z_{3}\right)}{2}+\frac{\left(z_{v}+\overline{z_{v}}\right)\left(z_{1}-z_{3}\right) e^{i \pi / 2}}{2\left(z_{v}-\overline{z_{v}}\right)} .
$$

The heading angle of the circle sector is $\theta_{c}=L\left[\left(z_{1}-\right.\right.$ $\left.\left.z_{3}\right) e^{i \pi / 2}\right]$. In the implementation $\theta_{c}$ is computed using the atan 2 function.

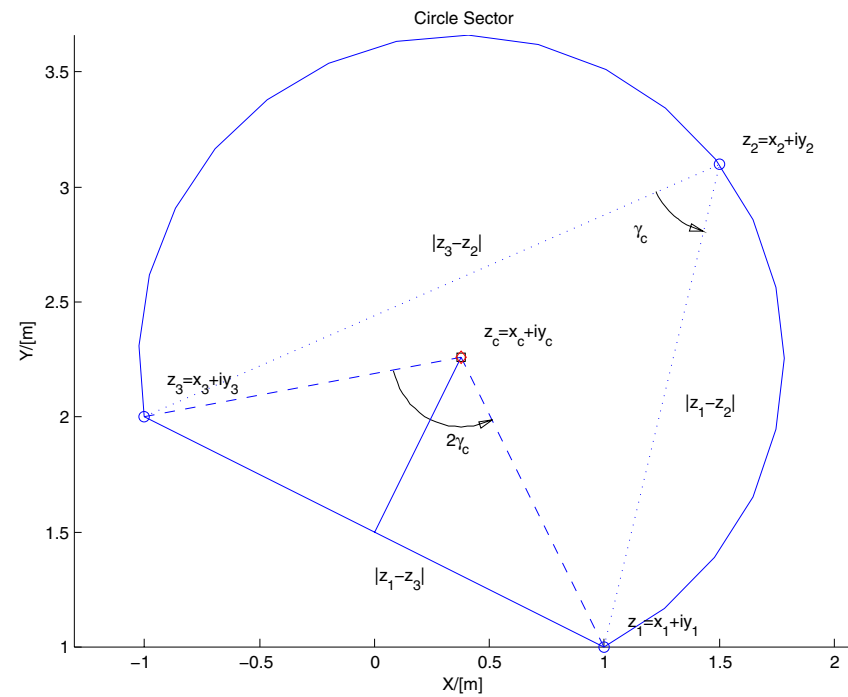

Fig. 5. The origin of the circle sector, $z_{c}$, can be calculated if the three bounding points are known. The theorem of inscribed angles in a circle is applied to get an expression for $z_{c}$.

The sector angle $\beta_{c}$ is calculated from the bisector angle, namely $\beta_{c}=2\left(\pi-\gamma_{c}\right)$. The radius $r_{c}$ for the circle bounded by $z_{2}$ is

$$
r_{c}=\sqrt{\left(x_{c}-x_{2}\right)^{2}+\left(y_{c}-y_{2}\right)^{2}} .
$$

In the expansion process the length of the chord, $\left|z_{1}-z_{3}\right|$, is compared to a threshold $t_{c h}$. If a chord is less than the threshold the expansion in corresponding direction is stopped.

\section{Collision-free path and safety margins}

Given a set of $\left\{\sec (1), \sec (2), \ldots, \sec \left(N_{\sec }\right)\right\}$ consecutive circle sectors, , has enough information to not only calculate a collision-free path, but also a left and a right safety margin for the vehicle. The set of circle sectors is used to calculate three polygons. One polygon is created by connecting the origins of the circle sectors,

$$
\operatorname{path}_{C}=\left[\begin{array}{llll}
x_{c}(1) & x_{c}(2) & \cdots & x_{c}\left(N_{s e c}\right) \\
y_{c}(1) & y_{c}(2) & \cdots & y_{c}\left(N_{s e c}\right)
\end{array}\right],
$$

which forms a collision-free path for the vehicle. One polygon for the left safety margin,

$$
\operatorname{margin}_{L}=\left[\begin{array}{c}
z_{c}(1)+\left(r_{c}(1)-m_{w}\right) e^{i\left(\theta_{c}(1)+0.5 \beta_{c}(1)\right)} \\
z_{c}(2)+\left(r_{c}(2)-m_{w}\right) e^{i\left(\theta_{c}(2)+0.5 \beta_{c}(2)\right)} \\
\vdots
\end{array}\right]^{T}
$$

and one safety margin to the right,

$$
\operatorname{margin}_{R}=\left[\begin{array}{c}
z_{c}(1)+\left(r_{c}(1)-m_{w}\right) e^{i\left(\theta_{c}(1)-0.5 \beta_{c}(1)\right)} \\
z_{c}(2)+\left(r_{c}(2)-m_{w}\right) e^{i\left(\theta_{c}(2)-0.5 \beta_{c}(2)\right)} \\
\vdots
\end{array}\right]^{T} .
$$

The desired safety margin, the distance to nearest obstacle, is denoted $m_{w}$. An example of calculated safety boundaries is visible in Figure 1 
Where the gateways to free-space are wide enough, the circle sector expansion continues. In a place where the expansion can proceed in two directions, the circle sector is divided into two parts, see Figure 6. Each part form a new circle sector and the possibility to pick a desired direction, either to the left or to the right. The radii of the unbounded sectors are limited to a default value.
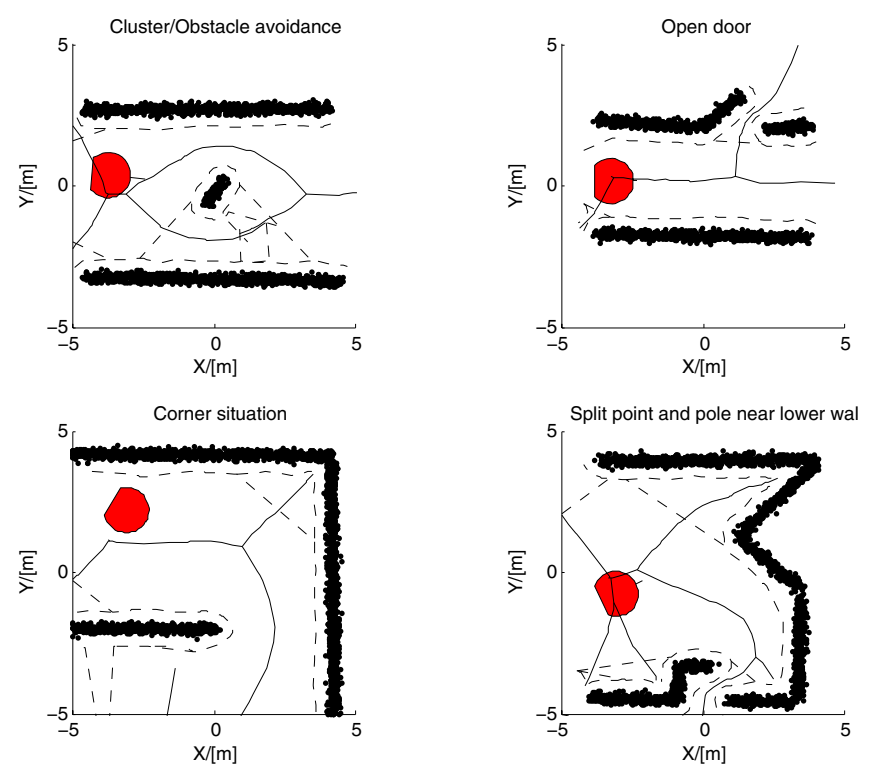

Fig. 6. Four examples of approximate Voronoi diagrams created by the circle sector expansion method. 1) Shows an example where an obstacle is present in a corridor. The method finds two possible paths for the vehicle to take. 2) A corridor environment with an open door. The method finds a path through the doorway. 3) The Voronoi diagram generated in a typical corner situation. 4) Shows a typical dead-end situation.

\section{E. Special cases of the CSE method}

There is a common interest to obtain obstacle avoidance in a realtime system [13]. The circle sector expansion has the advantage that it can perform obstacle avoidance from collected range data. The method returns a collision-free navigation area for the vehicle with safety margins included. In Figure 6 the safety margins are plotted as dashed lines.
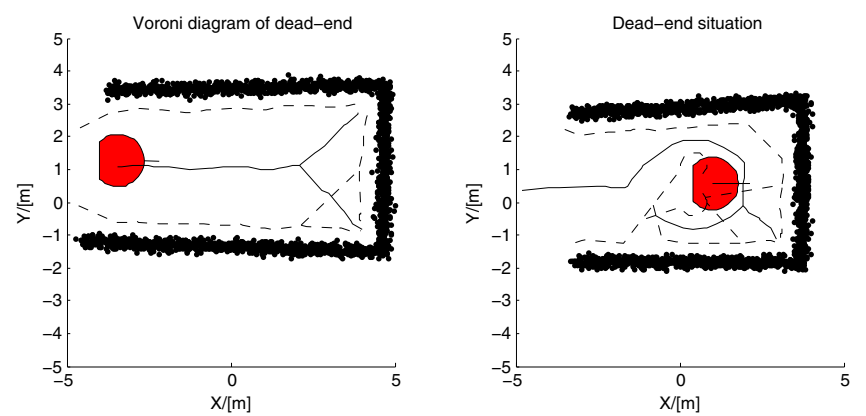

Fig. 7. A typical dead-end situation. The vehicle will in a normal situation get trapped in one of the corners. To deal with this problem the circle expansion method is used. Two possible return paths are found. The dashed line is the calculated safety margin for the robot.
If the robot reaches a dead-end situation, which means that the vehicle either must reverse or turn $180^{\circ}$, the solution is the following. As soon as a dead-end situation is identified (no further circle sector expansion is possible) the circle sector expansion restarts at the vehicle pose. The output is a possible path for the vehicle out of the trapped dead-end situation, as shown in Figure 7.

Some interesting properties of the CSE method;

- It is suitable for implementations on mobile robots.

- It reduces the number of points at the boundaries and the points needed to describe the available free space.

- The expansion process can start or continue at an arbitrary pose.

- It can be used to detect clusters of points. If a cycle is detected a cluster has been found and can be isolated. The points of a cluster can be sorted out by the surrounding polygon using the "Crossing Count" algorithm²[14]. The "Crossing Count" algorithm works as follows; given a point and a polygon the question is to decide wether the point lies inside the closed polygon.

- It can be used to find a path through a narrow passage such as a doorway.

- It can be used to determine if paths leads to dead-ends.

- It can be used to find paths around obstacles.

\section{IMPLEMENTATIONS AND TESTS}

This section is devoted to describing our test environment, the system built for the tests, as well as showing how our tests have successfully been performed. As is emphasized earlier in this paper; in addition to the theoretical proposal for solving the problem of exploration, we have also implemented and tested the circle sector expansion method. The CSE implementation can together with the MICA software be used on-line and is what we denote a soft real-time system[15].

1) Speedup of implementation: In the implementation the sector angle can be set to $\beta_{c}\left(1-\epsilon_{\beta_{c}} \sin \left(\beta_{c} / 2\right)\right)$ where $\epsilon_{\beta_{c}}$ is a small number. This will make the method faster to traverse data where the sector angles are close to $180^{\circ}$; such places are corridors.

2) Detection of a loop: Check if any of the previous found circle sectors, $\sec (1)$ to $\sec (k-1)$, is build of any combination of $z_{1}(k), z_{2}(k)$, and $z_{3}(k)$. Example; if the index numbers $673,741,981$ are found as combination $981,673,741$ a loop is detected. This will not work with the above described $\epsilon$ implementation.

\section{A. Hardware used in test}

The vehicle is a battery powered wheelchair which is equipped with sensors, a computer, and a wireless link to enable remote communication. It can either be locally, remotely controlled, or can work autonomously. It has wheel-encoders, one for each front wheel, for measuring the change in angle of the wheels. Its steering depends directly on the angular change of its front wheels, while its rear wheels are free and only

\footnotetext{
${ }^{2}$ http://www.visibone.com/inpoly/
} 


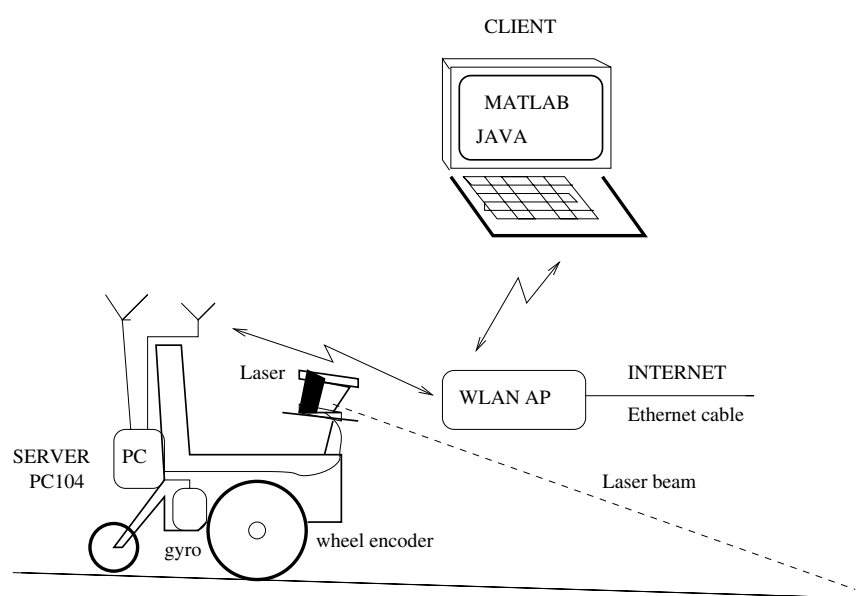

Fig. 8. The vehicle (a wheelchair) has an onboard PC104 based computer running Linux. The PC104 collects data from the gyro, ladar, and wheel encoders. It is connected to a wireless access-point (AP). A client computer can through socket communication connect to the server software and request sensor readings. The client computer runs MATLAB that process the sensor readings and computes control commands that are sent back the wheelchair. The laser is mounted on a pitched table. The laser beam hits the floor at a distance of approximately $4 \mathrm{~m}$ in front of the vehicle.

follows the movement given by the front wheels. The vehicle can continuously change its velocity and angular velocity. The width of the vehicle is that of an ordinary wheelchair, approximately $0.65 \mathrm{~m}$, which means that it can pass through regular doors.

It is also equipped with a rate gyro that, together with the encoders, is used for dead-reckoning of the vehicle pose.

The vehicle updates its position with a frequency of approximately $25 \mathrm{~Hz}$. The single-axis $\mathrm{KVH}$-Ecore 1000 rate gyro delivers data with a frequency of $10 \mathrm{~Hz}$.

The wheelchair has a range scanning laser for environment detection. It is positioned at a height of approximately $0.75 \mathrm{~m}$ from the floor and it is pitched down approximately $10^{\circ}$ and measures a sector of $180^{\circ}$, Figure 8 .

Each scan consists of 361 range data points that are transformed into 3D cartesian coordinates since the laser is tilted down. Due to the pitch, the forward sight of the laser is limited to $4 \mathrm{~m}$. Points within a distance $0.1 \mathrm{~m}$ from the ground are deleted in preprocessing of the range data.

\section{B. Start setting}

We consider a two-dimensional environment and a circle shaped vehicle with fixed radius. The start-up setting is such that the vehicle is standing still for some seconds while it scans its surrounding.

The initial pose of the vehicle $\left[x_{w}(0), y_{w}(0), \theta_{w}(0)\right]^{T}$ is $[0,0,0]^{T}$. The starting condition for the first circle sector is the vehicle pose, $x_{c}(1) \equiv x_{w}, y_{c}(1) \equiv y_{w}, \theta_{c}(1) \equiv \theta_{w}$, and $\beta_{c}(1) \equiv 1.5 \pi$. The start-up circle chord is defined by $z_{1}(1)$ and $z_{3}(1), z_{1}(1)=z_{c}(1)+r_{c}(1) e^{i\left(\theta_{c}(1)+\beta_{c}(1) * 0.5\right)}$ and $z_{3}(1)=$ $z_{c}(1)+r_{c}(1) e^{i\left(\theta_{c}(1)-\beta_{c}(1) * 0.5\right)}$. Using collected range data, a sequence of circle sectors are computed. In the circle sector expansion process the length of the chords are checked. The

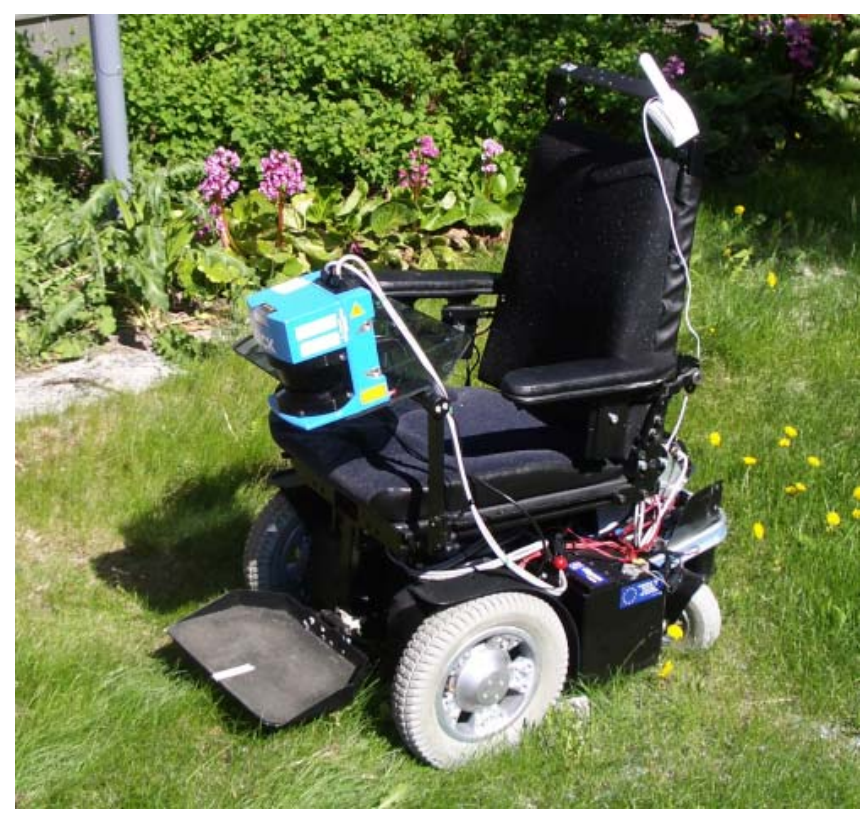

Fig. 9. The wheelchair used in the tests. It is equipped with a pitched range scanning laser, a rate gyro and two wheel-encoders. It carries an embedded x86 computer running RedHat Linux 7.3 which is used to collect and time stamp sensor readings. It controls the wheelchair. Clients can connect to the on-board computer over a wireless link and request sensor readings and send control commands. Using the implemented circle sector expansion method, the wheelchair was able to explore an unknown environment using the MATLAB environment on a standard Laptop computer running Windows-XP.

chord threshold is set to $t_{c h} \approx 0.8 \mathrm{~m}$, which is vehicle specific and means that found passages and free space is large enough for the wheelchair to reach. If the chord length is above the threshold the sector expansion process continues. From the computed consecutive circle sectors, polygons for the safety boundaries are calculated.

The margin $m_{w}$ is set to the $0.4 \mathrm{~m}$ to get obstacle clearance.

\section{The test environment}

Our tests are done in a part of the underground basement and corridors of Luleå University of Technology. The environment is a fairly general surrounding. It contains geometries such as straight corridors, crossing, and different kinds of obstacles with various shape complexity.

\section{Description of the on-line exploration}

On the client computer a background process reads position estimates and compares it against a desired path for the vehicle. The implemented path following algorithm can be found in [16] and [17]. After receiving commands from the control thread the vehicle starts to move. The circle sector expansion guides the vehicle through the environment. If computations become so slow that the vehicle is on the verge of colliding with obstacles an implemented watchdog function on the onboard computer detects it and automatically halts the vehicle. If no new driving commands are received by the onboard computer the wheelchair will automatically stop. The circle origins form a polygon for the vehicle to follow. The 


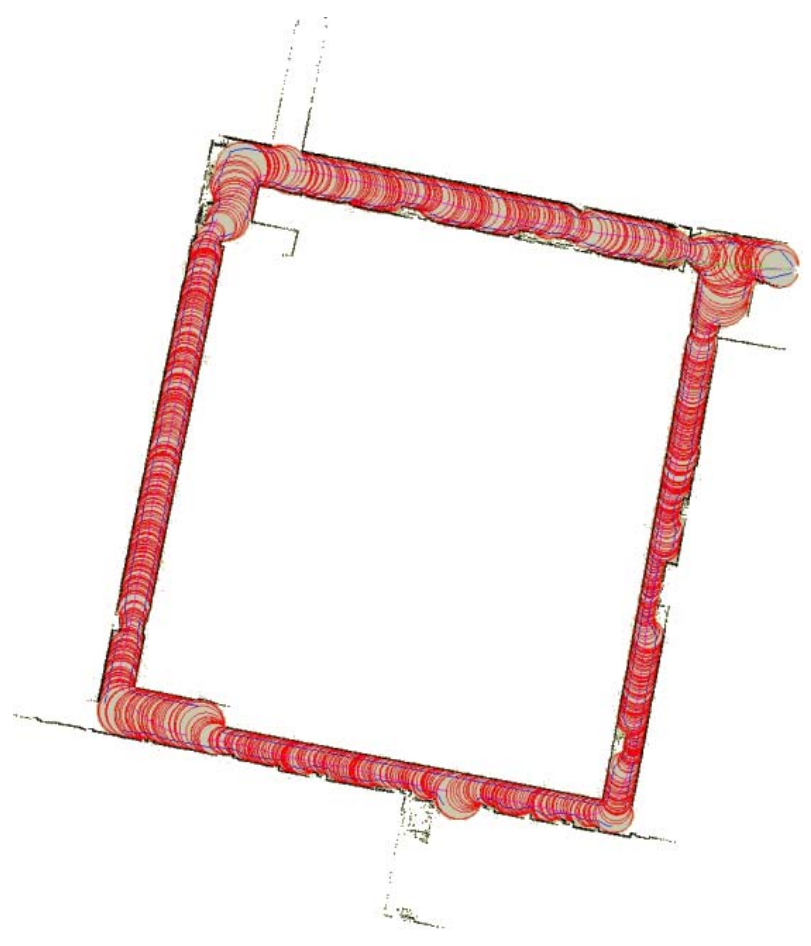

Fig. 10. Explored part of basement in the A-building at Luleå University of Technology. The upper left corner is outside room A1501 and the unexplored exit upwards is the passage that leads to the $\alpha$-building. The wheelchair moved in a clockwise direction. The circle sector expansion method was used to find a possible path and cover the present available free-space with consecutive circle sectors.

circle expansion gives the possibility to navigate and make a selection, go left, go right, follow the widest route, or follow the chord that leads towards a selected targeted position.

In exploration mode the circle expansion method is used to build a representation of the navigation area.

An example of a $2 \mathrm{D}$ environment that has been explored using circle sector expansion is shown in Figure 10 which is grabbed from the visualization implementation that uses the Open Scene Graph 3D engine [18]. The upper left corner of Figure 10 is visible in Figure 1. The exit upwards is a passage that leads from the A-building to the $\alpha$-building at Luleå University of Technology.

\section{CONCLusions}

We address the problem of driving an autonomous wheelchair in an unknown environment using a method based on circle sector expansions (CSE) on data collected by a range scanning laser. We propose an algorithm based on expanding circle sectors that calculates a reduced Voronoi diagram which is used for detecting available free-space. The CSE) method can handle corridors, split-points, corners, obstacles, clusters, and dead-ends. It is general and is suitable for the exploration of different kinds of unknown environments and not only for the particular test environment described in this paper.

The algorithm and its connected methods are intuitive and based on simple algorithms suitable for a vehicle motion in two dimensions. The method can be used for on-line obstacle avoidance and computation of safety margins. The implementation shows that the CSE method successfully works in practice. The main problems when implementing were spurioses that occurred on the wireless-LAN and sometimes the Java virtual machine ran out of memory.

However the final conclusion, with the wheelchair and its hardware and sensors in mind, is that the CSE method can be used to support wheelchair users in both unknown and known environments.

\section{ACKNOWLEDGMENT}

Thanks to Patrik Emilsson, working at Permobil ${ }^{3}$, for providing the wheelchair and his help with it.

\section{REFERENCES}

[1] S. Fortune, "A sweepline algorithm for voronoi diagrams," Algorithmica 2(2), pp. 153-174, 1987.

[2] P. Blaer, "Robot path planning using generalized voronoi diagrams," http://www.cs.columbia.edu/ pblaer/projects/path_planner/, Feb 2006.

[3] C. M. Gold and J. Snoeyink, "A one-step crust and skeleton extraction algorithm," Algorithmica, vol. 30, pp. 144-163, 2001.

[4] R. Ogniewicz and M. Ilg, "Voronoi skeletons: theory and applications," in IEEE Conference on Computer Vision and Pattern Recognition CVPR '92, June 1992, pp. 63-69.

[5] N. Rao, "Robot navigation in unknown generalized polygonal terrains using vision sensors," IEEE Transactions on Systems, Man and Cybernetics, vol. 25, no. 6, pp. 947-962, June 1995.

[6] R. Mahkovic and T. Slivnik, "Constructing the generalized local voronoi diagram from laser range scanner data," in IEEE Transactions on Systems, Man and Cybernetics, Part A, 2000, pp. $710-719$.

[7] T. Pendragon and L. While, "Path-planning by tessellation of obstacles," in Proceedings of Conferences in Research and Practice in Information Technology(ACSC'03), M. Oudshoorn, Ed., vol. 16, Adelaide, Australia, 2003.

[8] P. Beeson, N. K. Jong, and B. Kuipers, "Towards autonomous topological place detection using the extended voronoi graph," in IEEE International Conference on Robotics and Automation (ICRA'05), 2005.

[9] E. Acar, H. Choset, and J. Lee, "Sensor-based coverage with extended range detectors," IEEE Transactions on Robotics and Automation, vol. 22, no. 1, Feb 2006.

[10] O. Takahashi and R. Schilling, "Motion planning in a plane using generalized voronoi diagrams," IEEE Transactions on Robotics and Automation, vol. 5, no. 2, pp. 143-150, April 1989.

[11] G. Sakellariou, M. Shanahan, and B. Kuipers, "Skeletonisation as mobile robot navigation," in Towards Autonomic Robotic Systems (TAROS-04),, 2004.

[12] S. M. LaValle, Planning Algorithms. Cambridge University Press, 2006, to be published 2006.

[13] J. Borenstein and Y. Koren, "Real-time obstacle avoidance for fast mobile robots in cluttered environments 1," in IEEE International Conference on Robotics and Automation, 1990.

[14] H. Eric, Graphics Gems IV, P. Heckbert, Ed. Academic Press, 1994.

[15] S. Rönnbäck, D. Rosendahl, and K. Hyyppä, "A matlab/java interface to the mica wheelchair," in The 1st IFAC Symposium on Telematics Applications in Automation and Robotics, Helsinki and Finland, July 2004, pp. -

[16] J. A. da Cruz Pinto Gaspar, "Omnidirectional vision for mobile robot navigation," Ph.D. dissertation, UNIVERSIDADE T'ECNICA DE LISBOA,INSTITUTO SUPERIOR T'ECNICO, Dec 2002.

[17] C. udas de Wit, H.Khennouf, C.Samson, and O.J.Sordalen, Nonlinear control design for mobile robots, Nonlinear control for mobile robots. World Scientific series in Robotics and Intelligent Systems, 1993, ch. 5.

[18] OSG_Community, "Open scene graph, http://www.openscenegraph.org/," May 2005. [Online]. Available: http://www.openscenegraph.org/

\footnotetext{
${ }^{3}$ Permobil AB, http://www.permobil.se, June 2006
} 